\title{
Parking Lot Simulation by Discrete Event Model
}

\section{Tomohiro Fujimoto, Toshiharu Hatanaka and Katsuji Uosaki}

\author{
Department of Information and Knowledge Engineering, Tottori University,
}

Tottori 680 , Japan

\begin{abstract}
Due to rapid increase of vehicle use, construction of large scale parking lots is requested especially in urban area for smooth traffic. To improve the efficiency of available land use, some parking lots are designed as a hybrid type combining surface (flat) and tower parking. Design of such a kind of hybrid type parking is quite complex. Simulation methodology is useful to aid in design for such large scale parking lot. In this paper, a simulation method PLISM-1 based on discrete event model is developed with the concept of blocks and networks for solving this problem. By this simulation method, the evaluation of given parking lot plan can be done easily both from static and dynamic viewpoints.
\end{abstract}

Keywords. Simulation; parking lots; discrete event model; network; micro model; computer-aided-design.

\section{INTRODUCTION}

Recent increase of vehicle use requests the establishment of large scale parking lots, especially in urban area for smooth traffic. Hybrid type parking lots are developed by combining surface (flat) and tower parking to improve the efficiency of available land use. It is complex to design such a kind of hybrid type parking lots: where and how many surface and tower parking spaces are allocated; where the entrance and exit are allocated; what kind of through paths are established; what kind of guide system is effective; and so on, should be determined. We have no specific guidelines for this design and it is usually carried out by cutand-trial method. Given a parking lot layout and facilities plan, we evaluate its effectiveness and modify the plan based on the evaluation. Simulation is a useful tool to evaluate the parking lot plan. In this paper, we provides a simulation method based on discrete event model with the concept of blocks and networks to aid in the de- sign of parking lot facilities.

First, we give the description of the simulation model consisting of two parts; static and dynamic parts. The first part describes how we model the spatial characteristics of the parking lots and the second how we trace the vehicle movement.

We develop a simulation program PLISM-1 (Parking Lot Illustrative Simulation Method) to understand the characteristics of the given parking lot plan easily by observing the dynamic vehicle movement behavior on graphic display and evaluating efficiently several parking lot performance indexes, which are important for the design of parking lot facilities.

\section{SIMULATION MODEL}

We present here the simulation model. It is divided into two parts; static and dynamic parts. The spatial characteristics of the parking lots is given in the first part and the vehicle movement 
is described in the second part.

\section{Spatial Characteristics}

This part describes the spatial modeling of the parking lots. When a parking lot layout with twodimension spatial characteristics is given, we expand it into a set of blocks (segments) with onedimensional spatial characteristics (Fig.1) and construct their networks.

First we determine main line, which connects the entrance and the exit and most traffic flow goes through (under some guide system). Next, we find sub-lines, which branch off from or merge into the main line or other sub-lines. We call the branching points as intersections. Then we build up blocks.
They are composed of a line (main line or subline), parking spaces along the line and intersections. The block including main line is called as main block and that including sub-line is called as sub-block. Finally, we construct their network, which describes the linkage relation of the blocks including their hierarchy.

This segmentation is based on the observation that most parking lots employ one way system, and makes simulation model general but its implementation simple.

\section{Vehicle Movement}

In this part, how we trace the vehicle movement is described. We can write down the following
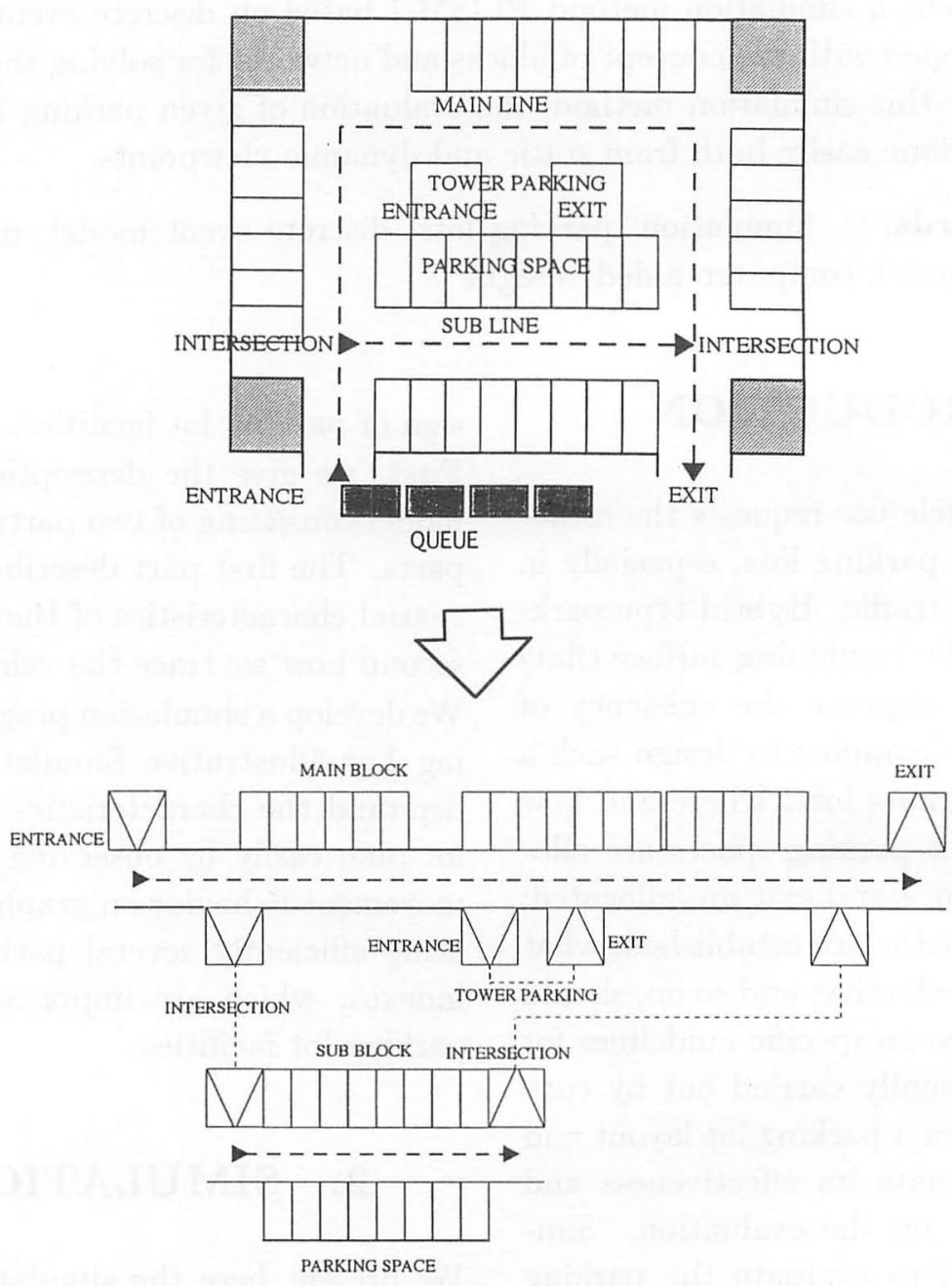

Fig.1 Segmentaion of the parking lot 
vehicle movement status in the parking system, some of which are shown in Fig.2.

1) Arrival at the parking lot

2) Entering the queue at the entrance

3) Leaving the queue at the entrance and entering the parking lot

4) Running on the road

5) Waiting on the road due to parking procedure or attempting to leave the current block by the preceding vehicle

6) Waiting at the intersection for attempting to leave the current block

7) Leaving the current block and entering another block through intersection

8) Queue in front of the target parking space

9) Parking procedure into the parking space

10) Parking duration in the parking space

11) Waiting for unparking

12) Unparking procedure from the parking space

13) Leaving the parking lot through the exit

We define the events. Here, the entrance, parking spaces, intersection and the exit are called generally as points.
1) Arrival of the vehicle - Vehicle arrives at the parking lot entrance. This event includes 'arrival at the parking lot' according to some probabilistic law such as Poisson process, and 'entering and leaving the queue at the entrance'.

2) Proceeding to the target point - Vehicle proceeds to the target destination point. This event includes 'running on the road', 'parking procedure into the parking space', 'parking duration', 'unparking procedure from the parking space' and 'leaving the parking lot through the exit'. When there is some obstacles such as a vehicle in front of it attempting parking or leaving the current block through intersection, it waits on the road (line) until the obstacle conditions are removed (see, for example, Fig.3). Time for parking and unparking procedures, speed of the vehicles and parking duration time are given in advance. For parking, the destination point is given when the vehicle enters the parking lot.

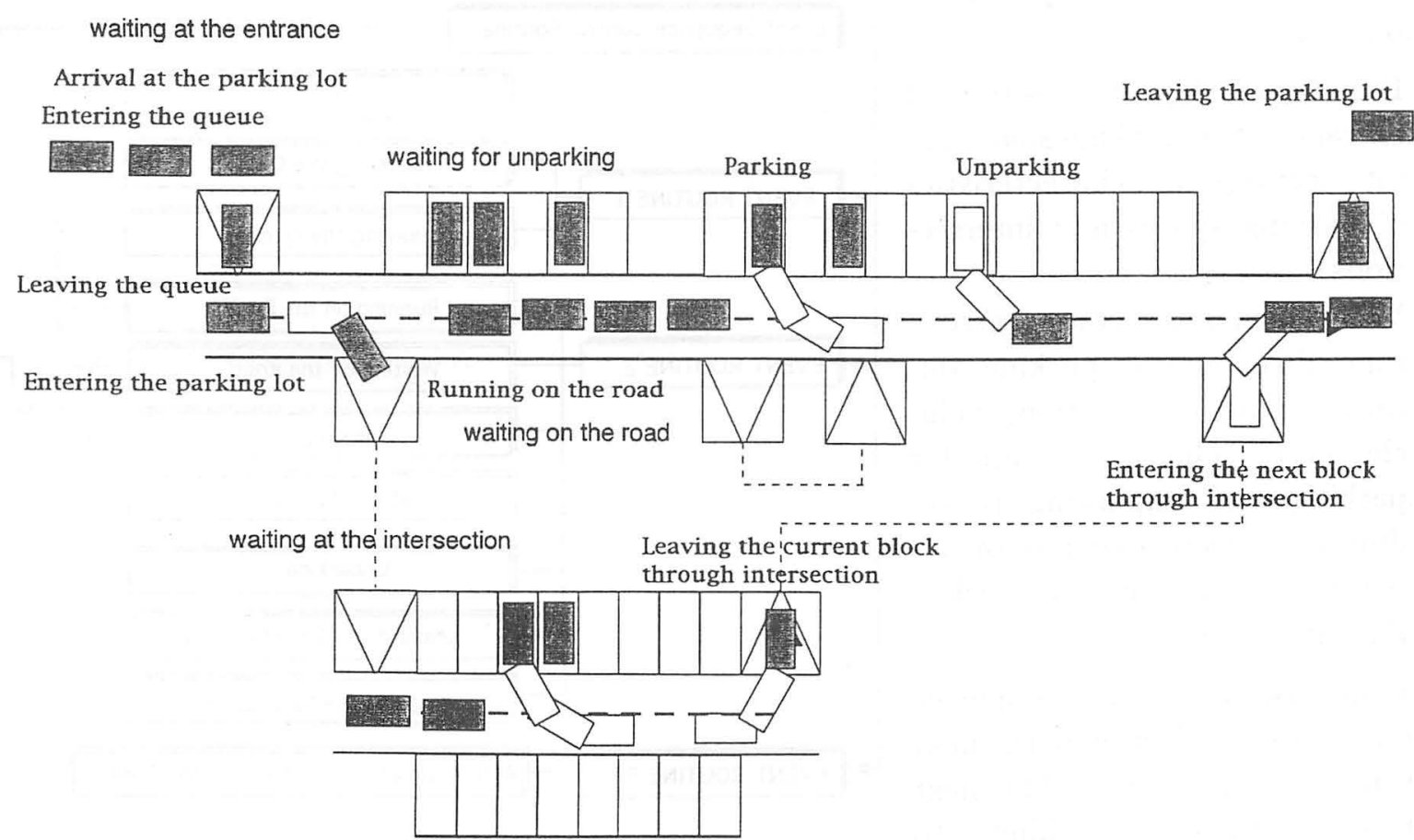

Fig.2 Vehicle movement status in the parking lot 
3) Attempting to leave the current point - Vehicle attempts to leave the current point. This event includes attempting 'unparking' after parking duration, 'leaving the parking lot', and 'leaving the current block and entering another block through intersection'. The vehicle looks for an appropriate gap to enable it to unpark or to enter another block through intersection.

\section{SIMULATION PROGRAM}

Based on the model description in the preceding section, we have developed a simulation program PLISM1. It contains an event sequence control routine and three event routines as shown in Fig.4. The event sequence control routine starts the simulation by receiving the input information such as

1) Parking lot layout (position of entrance, exit, parking space and intersections, and characteristics of parking spaces and intersections)

2) Vehicle characteristics (arrival time distribution, parking duration time distribution, vehicle speed, vehicle size, time for parking and unparking procedure and acceptance gap for unparking or entering new block)

3) Executing time

The main task of the event sequence control routine is selection of the next event to be carried out. The next event is determined by taking into the account of the occurrence time of the events for all the vehicles in the parking lot and the arrival time of the

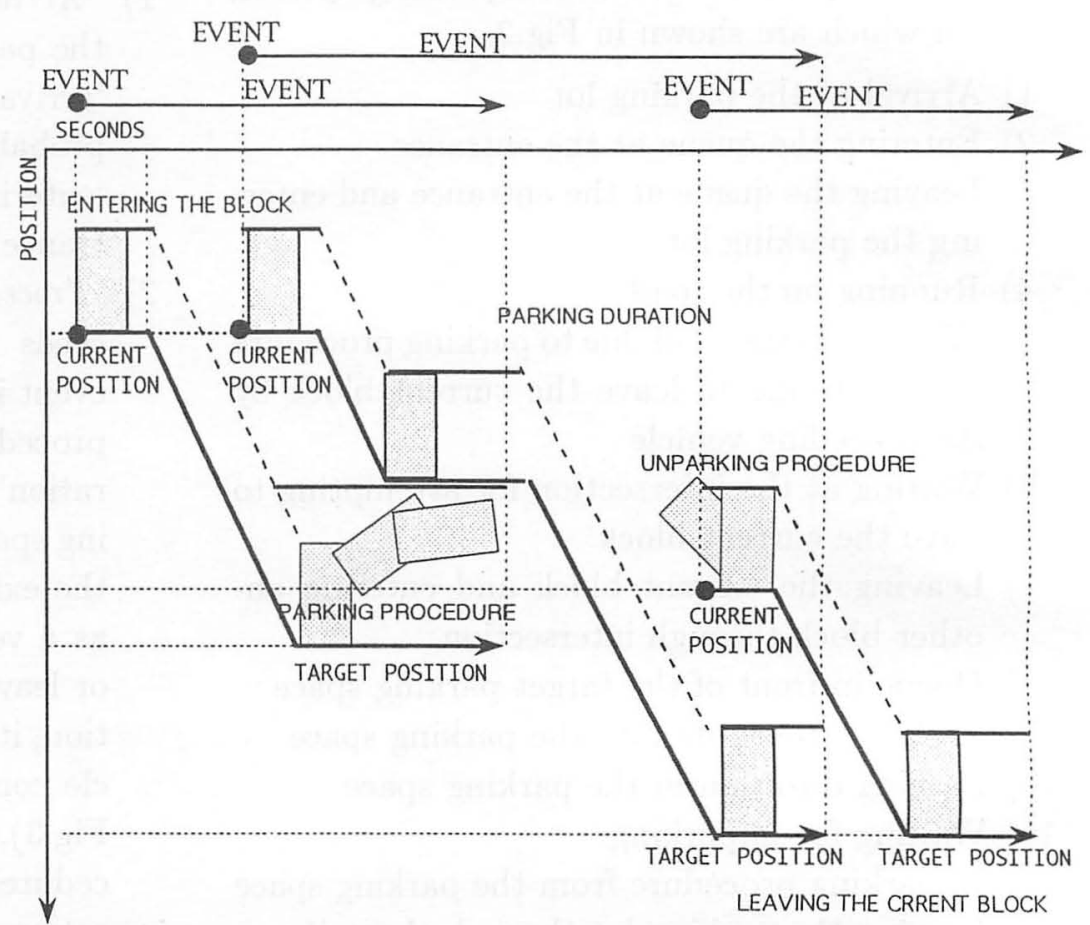

Fig.3 Space-time diagram and obstacles on the road

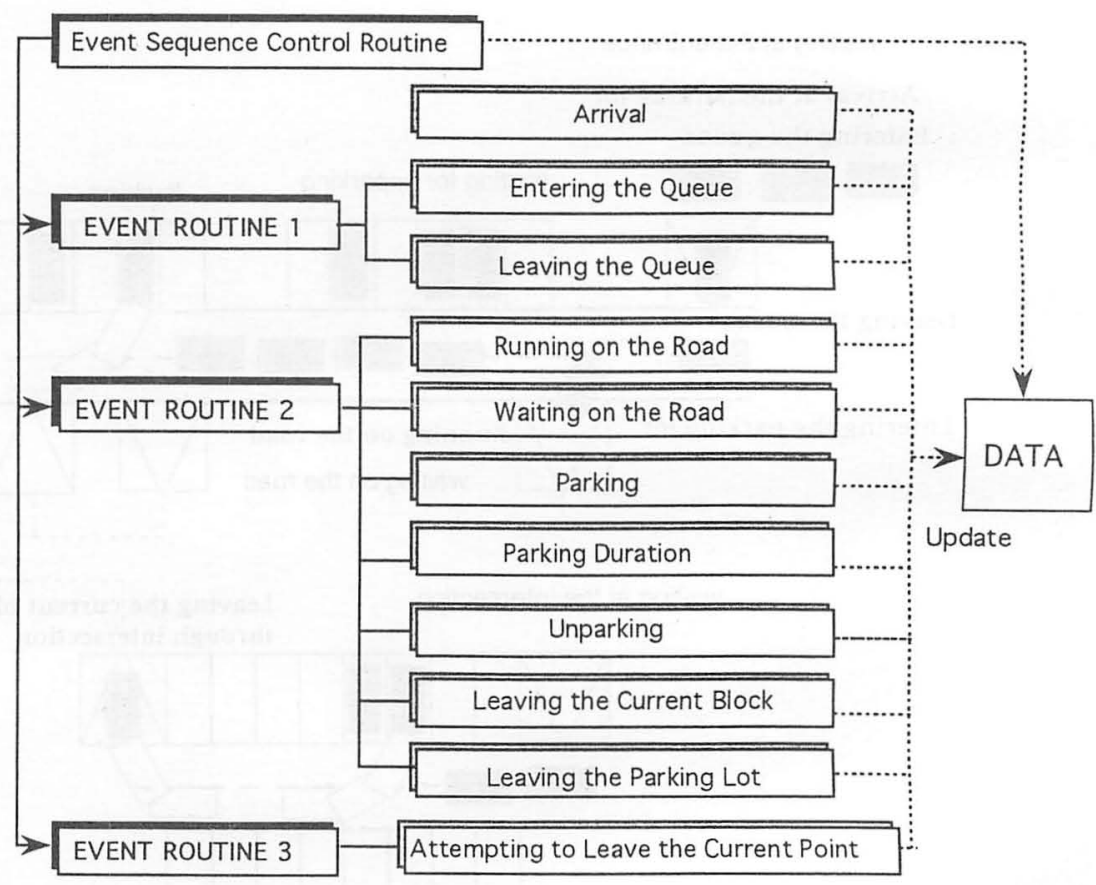

Fig.4 Block diagram of PLISM-1 
next vehicle. The occurrence time of each event is calculated analytically or given in advance. Event routines 1,2 and 3 execute the events arrival of the vehicle, proceeding to the target point, and attempting to leave the current point given in the preceding section, respectively.

Information on arrival time, exit time, spatial position, parking, unparking and parking duration for every vehicle moving through the parking lot system are filed out and can be observed as in Fig.5. From this file, we can evaluate efficiently several important parking lot performance indexes such as
1) Utilization rate of the parking lot

2) Queuing time outside the parking lot

3) Loss time on the lines in the parking lot

4) Maximum queue length at entrances and exits

5) Mean queue length at entrances and exits

The output of PLISM-1 provides the vehicle position in the parking lot (Fig.6). The displaying this information over time enables us to observe the dynamic display of the vehicle movements. This display is of considerable aid in evaluating the parking lot layout by seeing the places queue often occurs, for example.

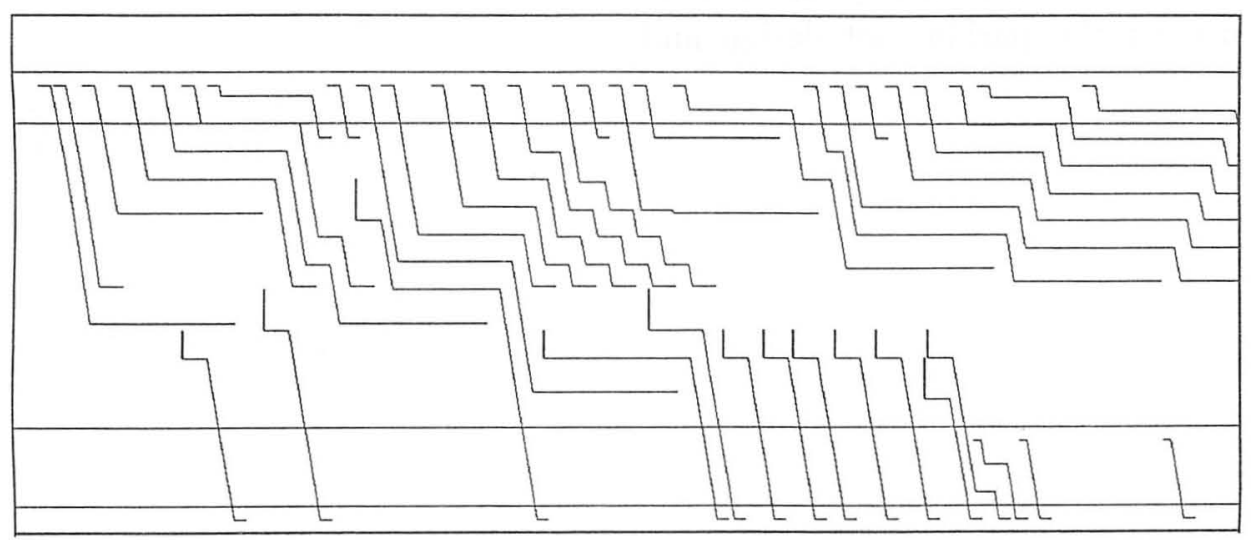

Fig.5 Trace of vehicle movements

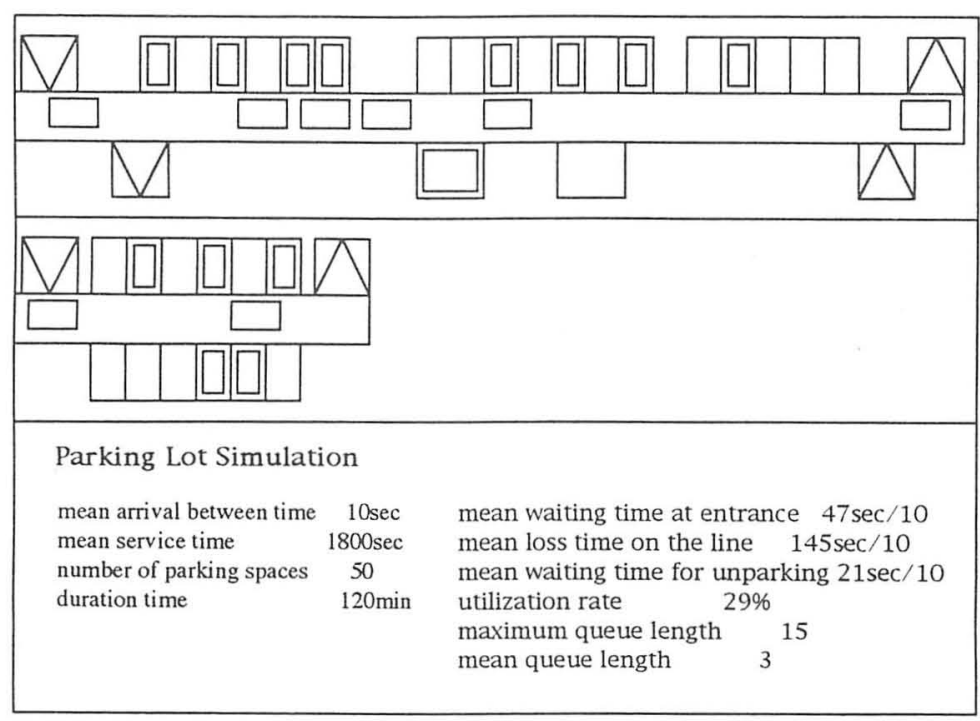

Fig.6 Simulation results on the display 


\section{CONCLUSIONS}

We have developed a simulation program PLISM1 based on discrete event model with the concept of blocks and networks to aid in the design of parking lot facilities. We approximate two-dimensional spatial characteristics of the parking lot by a set of one-dimensional blocks and their networks, and then apply the concept of discrete events. By this modeling, we can simulate the vehicle movement in the parking lot efficiently. The simulation program PLISM-1 provides the dynamic display of the vehicle movements in addition to several parking lot performance indexes. This program is of considerable use for the parking lot design and evaluation.

\section{REFERENCES}

1) S. Miano and A. Eiger, Parking lot design by interactive computer graphics, Computer and Graphics, 5, 115-121 (1980)

2) T. Nakanishi, Computer Simulation, KindaiKagaku (1977)

3) W. Young, Vehicle movements in parking facilities, Mathematics and Computers in Simulation, 28, 305-309 (1986)

4) W. Young, Parking Systems Modelling, MS Thesis, Department of Computer Sciences, Monash University (1989) 